\title{
RESEARCH ON THE INTEGRATION OF PDM AND SOLIDWORKS
}

\author{
Danqing Zhu' ${ }^{1}$, Dong Yan ${ }^{2}$ \\ ${ }^{1}$ College of Mechanical Engineering, Shanghai University of Engineering Science, China \\ ${ }^{2}$ College of Mechanical Engineering, Shanghai University of Engineering Science, China
}

\begin{abstract}
The integration of CAD system and PDM system is the most difficult part in the process of PDM system integration, and its key is to ensure the consistency of data changes betweenCAD system and PDM system. This paper introduces how to integrate SolidWorks in PDM system to achieve the automatical establishment of BOM table.
\end{abstract}

Keywords: - PDM; SolidWorks; API

\section{INTRODUCTION}

PDM(product data management) is a tool that helps engineers and others manage product data and product development processes.It can integrate CAD, CAE, CAM, CAPP, etc., which is a core part of CIMS(computer integrated manufacturing system).SolidWorks is an excellent $3 \mathrm{~d}$ mechanical design software based on Windows platform. Its parameterization and feature modeling technology provide a good design environment for designersand is widely used in enterprises. To manage the information of SolidWorks through PDM, the integration of SolidWorks and PDM is required by the enterprises.

$\mathrm{BOM}(\mathrm{BOM})$ is the product structure data file which can be recognized by computer and the carrier of product data transmission throughout the life cycle.BOM runs through product concept design, calculation analysis, detailed design, process planning, prototype trial manufacture, processing and manufacturing, sales and maintenance until the product dies.In PDM, BOM technology is the core, and the success or failure of a PDM system depends largely on the quality of BOM technology. In this paper, we will discuss how to automatically establish BOM table in PDM system and the integration between PDM system and Solidworks.

\section{INTEGRATION MODE OF PDM SYSTEM AND CAD SYSTEM}

The integration of CAD system and PDM system is the most difficult part of PDM system. The key of this issue is to ensure the consistency of data transition between CAD system and PDM system. There are three integration modes between PDM system and CAD system:

(1) Encapsulation: In this mode,CAD system and PDM are basically separated, and the system user needs to manually enter the attributes of CAD files and manually set up the information required in the BOM table in the PDM system. This method is of poor integration, low automation, complicated operation and inconvenient use.
(2) Interface type: This pattern can only be achieved after the program has been second developed.Users can write interface program by using CAD API function to get products directly within the assembly relation between the zero parts and components of relevant information, and then spread to the product data management (PDM), automatically add to the BOM of product data management (PDM), coexist to relevant database storage preservation. At the same time, the latest structural relation can be extracted from the BOM of PDM, which is used to modify the assembly files of $\mathrm{CAD}$, so as to keep the information consistent between the systems. This approach has a high degree of integration and automation, but it has a compatibility problem with program development.

(3) Tight integration mode: This pattern is the highestlevel set.It is a highly integrated mode for both PDM and CAD systems to provide two-way exchange and shared information for all types of information, such as product data, feature data and application data. This kind of integrated automation degree is the highest, the program compatibility stability is good, but the development is more complex and can only manage to the specific CAD system. Such as SolidWorks company's own distributed PDMWorks software.

\section{THE COMMON PROBLEM OF SYSTEM INTEGRATION}

In the past year, the author has visited more than 20 enterprises that have implemented SolidWorks 3d CAD system and product data management system, and conducted surveys on IT, R\&D and management departments respectively. It was found that the problems caused by imperfect system integration were mainly as follows.

(1) The product upgrade interface needs to be redeveloped. After enterprise deployed SolidWorks and product data management (PDM) system, the interface was developed through service provider, and because of the high cost of maintenance and staff turnover, the interface lackssustained protection, unable to support the upgrade 
of $3 \mathrm{~d}$ CAD system and product data management system, cause that enterprise cannot used improved IT system to better serve the R\&D department.

(2) Manage $R \& D$ release data only, not process data.In these enterprises, the integration interface is used only as a data archive.The projectdocuments are still saved in the local computers in the dailyprocess and a large amount of design process data is easy tolost, the design experience cannot beaccumulated, and the process analysis and improvement and design retrospection cannot be carried out.In the end, when we do design reviews, we just know that the results are not correctbut we don't know where the problem is.

(3) Complex integration operation and low application efficiency."We all work for PDM, but it doesn't help us with our work," one of the engineers in the study was quoted as saying. Although this sentence is a bit extreme, it also reflects the resistance of engineers caused by the complex interface application.The complex operation and low application efficiency are mainly reflected in the frequent switching of different software in the design process of engineers. One is in the setting of CAD system, the other is in the search of product data management system (PDM), the results are downloaded to local computer, and then open it in the CAD system.Product deformation design need download the entire model, it is a total waste of time and laborious.Therefore, among these customers, engineers complain about the PDM system, and the benefits of product data management systems such as coordination, sharing and efficiency, which are emphasized in the selection process, are not reflected.

(4) The complete engineering BOM information cannot be extracted.In the original concept,when engineers extract engineering BOMs, which are usually based on twodimensional CAD system, they only need to consider the structure information extraction, it cause that the SolidWorks assembly model and zero model, based on different design methods and application of BOM information for part of the project, can't be extract, such as virtual models, multiple configuration list, welding and pipe, etc.Therefore, it is impossible to extract the complete engineering BOMinformation, and needs to be processed manually in the later process, which is inefficient and prone to errors.

(5) The model and engineering drawing cannot be synchronized. When the product data is managed in the product data management system (PDM), the $3 \mathrm{~d}$ model is not associated with the engineering drawings, resulting in the document being unable to be synchronized, and requires manual processing, brings inconvenience to the daily processing of engineers and is prone to errors.

\section{THE REQUIREMENTS OF INTEGRATION BETWEEN SOLIDWORKS AND PDM SYSTEM}

With the continuous progress of enterprises in the design and innovation field, more and more complex requirements have been put forward for $3 \mathrm{~d} C A D$ system. The $3 \mathrm{~d} C A D$ system has also gradually changed from tool level to system level.SolidWorks, as the most popular 3d CAD system in the engineering equipment industry, releases new version every year for continuous improvement and improvement to meet customer needs.From the first SolidWorks released to the market in 1995, there were 26 official releases by 2018.There are hundreds of feature improvements and upgrades for each official release.SolidWorks is also changing from a simple $3 \mathrm{~d}$ structure design software to one used in multidisciplinary and multi-specialty complex product research and development.The continuous development of this $3 \mathrm{~d}$ CAD system also brings difficulties to its integration with product data management system (PDM).

When customers have already used SolidWorks 3d CAD system and need to deploy product data management system (PDM), they need to take full account of the existing SolidWorks 3d CAD system and sort out the integration requirements.Basedon the previous research and experience accumulated during the implementation of a large number of projects, the author summarized the integration needs of customers into the following aspects.

\subsection{Support the Collaborative Design of SolidWorks 3d CAD System}

In the support requirements of system integration for collaborative design, the main emphasis is on data exchange and timely data update in the design process.In daily product design, engineers use most of the time on $3 \mathrm{~d}$ CAD system and various design tools. In the research, we found that almost all the engineers hope to access and share the needed data in the 3D CAD system in order to timely understand the data changes from other personnel of the working team, and be able to communicate and exchange with other personnel.

According to the above requirements, we can summarize the integration requirements for collaborative design into the following points :(1) to search and share design data within design tools such as SolidWorks 3d CAD system;(2) the design data can be locked and updatedthrough the control mechanism;(3) can quickly update existing data in SolidWorks 3d CAD system and inform relevant personnel;(4) real-time and dynamic understanding of product related design data and timely design adjustment;(5) communicate with other designers when necessary, communicate based on text and visualization, and understand design intention;(6) when necessary, carry out cross-departmental and professional cooperation, such as transferring the model to the analysis department or other collaborative departments. Through the above requirements, an online design model based on a single data source can be formed to enhance design coordination within the department and across departments, avoid unnecessary mistakes in the process, and strengthen team communication in the design process. 
Through the above requirements, an online design model based on a single data source can be formed to enhance design coordination within the department and across departments, avoid unnecessary mistakes in the process, and strengthen team communication in the design process.

\subsection{Support the Process Management of Design}

\section{Data}

For the process management of design data, it should support not only the archival management of released data, but also the design data management in the design process.In the process of product design, especially in the process of new product design, the data generated by the whole design team has to go through a long process. Thedesign data of product model, simulation data, various technical specifications, etc. all need to record the baseline of the whole process from generation to release.These process data can not only help engineers to carry out the necessary design backtracking, but also can coordinate the design process data to improve the parallel design efficiency.In addition, the data of the whole design process is also the precipitation of the design experience of enterprises. In the R\&D innovation enterprises, the design process is more valuable than the design result: with the continuous improvement of the R\&D innovation ability, the design process needs to be continuously analyzed and improved.Therefore, the control of the design process is crucial to the enterprise.To summarize the requirements of the enterprise in the process management of design data, the following are the main points :(1) update and trace the process data in the design environment, and simplify the operation;(2) bi-directional mapping can be carried out at the data level. For example, changing parameters in PDM can drive model or model parameters or change design model to make new parameters in PDM;(3) data generation to release data, especially baseline data can be fully recorded without affecting system efficiency;(4) not only record the modeling process data, but also include modelbased simulation and other data;(5) be able to handle the intricate data correlation in the design process;(6) process data should include the collaborative process of data, including review and verification, etc.

Through the above requirements, the management and precipitation of enterprise design process data can be realized, and the product data management system (PDM) becomes the database of enterprise design knowledge.

\subsection{The Capability of Complete Extraction of Engineering BOM Information from the Model}

In the application of product data management system (PDM), it is one of the basic conditions to extract and manage the engineering BOM information contained in $\mathrm{CAD}$ system. However, many enterprises often ignore the requirement to extract engineering $\mathrm{BOM}$ information in SolidWorks $3 \mathrm{~d}$ CAD system when selecting the original
model.With the improvement of enterprise design methods, more requirements on engineering $\mathrm{BOM}$ extraction have been put forward.These information will be stored in different forms according to different customers' applications. Generally, when integrating with SolidWorks $3 \mathrm{~d}$ CAD system, the extraction of the following common information needs to be taken into full consideration: (1) virtual part information extraction in SolidWorks model;(2) extraction of welding list information in the model;(3) multi-configuration signal extraction in SolidWorks model;(4) pipeline information extraction in SolidWorks model.Only by fully considering the information extraction required by engineering $\mathrm{BOM}$ can the product data management system (PDM) be able to manage the complete engineering BOM information of products and provide the required data to the back-end interface system or department.

\subsection{Support based on SolidWorks 3d CAD System}

In the current SolidWorks three-dimensional CAD system, there is a large number of existing design libraries, which facilitates engineers to make rapid calls on some models and accelerate product design in the design process. This is also the characteristics and advantages of SolidWorks system, which is one of the requirements of customers when they first choose SolidWorks products.The product data management system (PDM) needs to take into account the inheritance and management of these valuable design libraries when it is deployed to avoid a lot of library transformation and reconstruction.In SolidWorks 3d CAD system, the following design libraries are mainly included :(1) ToolBox standard parts library;(2) CircuitWorkscomponent library;(3) simulation analysis library;(4) Routing part library.If these $3 \mathrm{~d}$ design libraries cannot be inherited and managed well, the application efficiency of SolidWorks system will be greatly reduced, and the investment rate of original investment and purchasing $3 \mathrm{~d}$ system will be reduced.5. Support the extended application of SolidWorks 3d CAD system based on SolidWorks. SolidWorks 3d CAD is gradually transformed from a single structural design tool to a multidisciplinary design integration system. There are certain data interchange and system integration between these tools, and they cover different departments in the enterprise R\&D organization. They also have higher requirements for the integrated application of product data management system (PDM). In the overall solution, we need to integrate and manage the following extended systems :(1) Electrical design;(2) Simulation analysis;(3) production of technical exchange documents of Composer.By integrating different systems in the above SolidWorks $3 \mathrm{~d}$ digital solutions, enterprise data management will besmoother and integration is closer.We will truly establish a multidisciplinary, multiorganizational and multi-regional $\mathrm{R} \& \mathrm{D}$ and innovation platform for enterprises. 


\section{METHOD OF INTEGRATION IMPLEMENTATION OF PDM SYSTEM AND SOLIDWORKS SYSTEM}

\subsection{Issues}

SolidWorks is a Windows based CAD/CAE/CAM's desktop operating system, PDM, as a platform for enterprise product development management, needs to integrate information from different systems to realize the management of corresponding documents and data.This paper uses the selfdeveloped Skywork PDM system, aiming at the diversity of CAD software used in the enterprise, adopts the second kind of integration and discusses how to extract the BOM table information in SolidWorks.

\subsection{Integration Conditions}

\subsubsection{Selection of Integration Environment}

SolidWorks offers a wide range of OLE objects, and these methods and properties of as have, users can use it and through the support OLE programming development tools, such as vc++ 6.0, VB and VBA tool for secondary development, through in the customer's application for the OLE object and its methods and properties of the operation, can be realized in the development of application software of SolidWorks software almost all the functions, establish suitable for SolidWorks function modules, dedicated to meet the needs of users.

\subsubsection{The API Objects in SolidWorks}

Figure 1 is the SolidWorksAPI object relational hierarchy diagram. SolidWorks software provides a complete, free secondary development tool :SolidWorks API(application programming interface). SolidWorks API and its related documents are included in SolidWorks software, and any user can make a secondary development of SolidWorks. SolidWorks API is the OLE programming interface of SolidWorks, which provides a fully object-oriented class system for programmers.

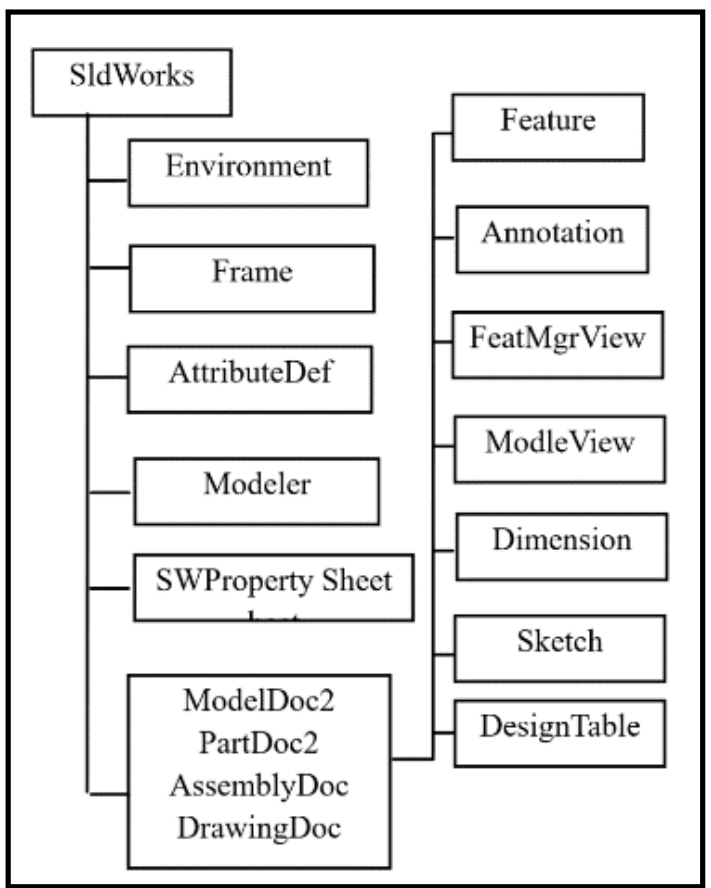

Fig 1: The SolidWorksAPI object relational hierarchy diagram

\subsection{Implementation of Integration Functions}

(1) Open Class in VC++6.0 engineering files wizard selects the "From type library" option in Add Class.

(2) Open in the "Import from type library" dialog boxSldwork.lib file, which is placed in the installation directory of SolidWorks.

(3) Directly select the required class object in the confirm classes dialog box, confirm and join the project.

(4) Write code implementation functions with added classes.

The following is the implementation of the SolidWorks file extracted from the project:

sldObject.CreateDispatch("SldWorks.Application"); sldObject.OpenDoc6 ( sFileName3, 0, " ",\&nErrors, \&nWarnings) pDispatch=sldObject.GetActiveDoc() pDrawingDoc.AttachDispatch(pDispatch); if(!pDrawingDoc)return; pDispatch=pDrawingDoc.GetFirstView(); pView.AttachDispatch(pDispatch); pDispatch $=$ pView.GetBomTable () while( !pDispatch ) \{ pDispatch $=$ pView.GetNextView () ; pView.AttachDispatch(pDispatch); pDispatch $=$ pView.GetBomTable () ; \} pBomTable.AttachDispatch(pDispatch); ral = pBomTable.Attach3 (); 


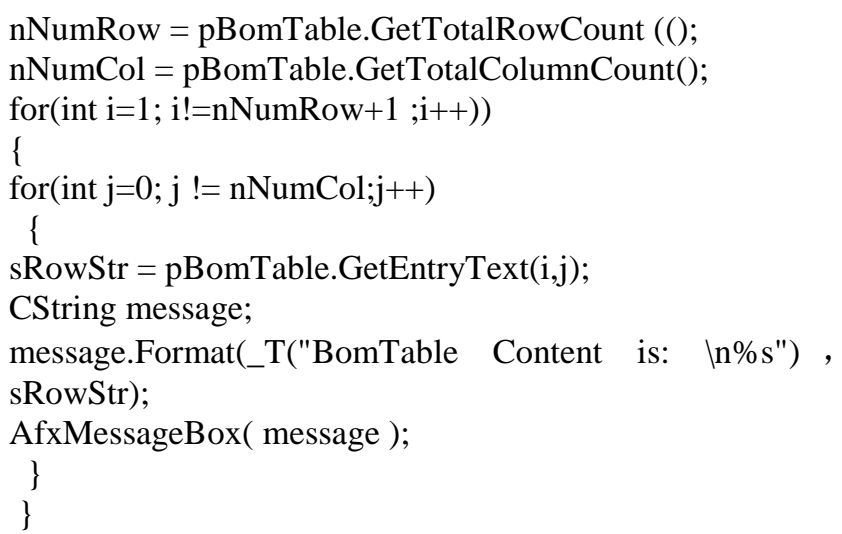

Through these codes, BOM information can be read into the project, which guarantees the consistency of data between PDM system and SolidWorks. The integration of PDM and SolidWorks can be achieved by completing the storage of database through other code.

\section{CONCLUSION}

PDM product data management software, as an integrated platformand frameworks, needs a lot of software integrations to implement information management.In this paper, BOM information extraction is taken as an example in the integration process of Skywork PDM and SolidWorks software, and the key technologies and methods of integration are introduced.

\section{REFERENCES}

[1] MaShengxiang, Yi chuanyun, Li Ming, second development research of SolidWorks [J]. Mechanical engineer, 2005.

[2] Wu Yun, Shang Fengwu. Discussion on BOM table in PDM application system integration [J]. Journal of engineering graphics, 2002.

[3] Liu Shunshu, Zhu zhaoguang. Integrated design of SolidWorks system and PDM system [J]. Modern manufacturing engineering, 2004. 